\title{
Development and Performance Verification of Fiber Optic Temperature Sensors in High Temperature Engine Environments
}

\author{
Grigory Adamovsky ${ }^{1}$ \\ NASA Glenn Research Center, Cleveland, $\mathrm{OH} 44135$ \\ Jeffrey R. Mackey ${ }^{2}$ and Lawrence A. Kren ${ }^{3}$ \\ Vantage Partners, LLC, Brook Park, OH 44142 \\ Bertram M. Floyd ${ }^{4}$ \\ Sierra Lobo, Inc., Cleveland, OH 44135 \\ Kristie A. Elam ${ }^{5}$ \\ Jacobs Technology, Cleveland, $\mathrm{OH} 44135$ \\ and \\ Martel Martinez ${ }^{6}$ \\ NASA Armstrong Flight Research Center, Edwards, CA 93523
}

\begin{abstract}
A High Temperature Fiber Optic Sensor (HTFOS) has been developed at NASA Glenn Research Center for aircraft engine applications. After fabrication and preliminary in-house performance evaluation, the HTFOS was tested in an engine environment at NASA Armstrong Flight Research Center. The engine tests enabled the performance of the HTFOS in real engine environments to be evaluated along with the ability of the sensor to respond to changes in the engine's operating condition. Data were collected prior, during, and after each test in order to observe the change in temperature from ambient to each of the various test point levels. An adequate amount of data was collected and analyzed to satisfy the research team that HTFOS operates properly while the engine was running. Temperature measurements made by HTFOS while the engine was running agreed with those anticipated.
\end{abstract}

\footnotetext{
$d_{l} \quad=$ Outer diameter of a smaller diameter ceramic tube

$d_{2} \quad=$ Outer diameter of a larger diameter ceramic tube

$d n=$ infinitesimal change in the refractive index

$d T=$ infinitesimal change in temperature
}

${ }^{1}$ Sr. Research Engineer, Optics and Photonics Branch, MS 77-1, 21000 Brookpark Rd. Cleveland, OH 44135, AIAA Associate Fellow.

${ }^{2}$ Optical Research Engineer, GESS-3, 3000 Aerospace Parkway, Brook Park, OH 44142.

${ }^{3}$ Mechanical Engineer, GESS-3, 3000 Aerospace Parkway, Brook Park, OH 44142.

${ }^{4}$ Lead Engineering Technician, MS 77-3, 21000 Brookpark Rd. Cleveland, OH 44135.

${ }^{5}$ Optics Technician, MS 77-3, 21000 Brookpark Rd, Cleveland, OH 44135.

${ }^{6}$ Engineer, Operations Engineering Branch, MS 4820/200B, PO Box 273, Edwards CA 93523. 


$\begin{array}{ll}N & =\text { number of grating line spacings in a grating } \\ n & =\text { refractive index } \\ P_{\mathrm{i}, \mathrm{j}} & =\text { Pockel's coefficient of the stress-optic tensor } \\ \alpha & =\text { coefficient of thermal expansion of the fiber material } \\ \Delta n & =\text { longitudinal periodic variation of the refractive index of the optical fiber core in a grating } \\ \Delta T & =\text { temperature change } \\ \Delta \lambda & =\text { full-width-half-maximum bandwidth of a grating } \\ \Delta \lambda_{B} & =\text { shift in the Bragg wavelength due to temperature and pressure } \\ \varepsilon & =\text { applied strain } \\ \Lambda_{G} & =\text { grating period } \\ \lambda_{G} & =\text { Bragg wavelength } \\ v & =\text { Poisson's ratio }\end{array}$

\section{Introduction}

$\mathrm{G}$ obal commercial aviation traffic is projected to exhibit continued future growth. While commercial aviation travel is extremely safe, if accident rates are not reduced there will be an increase in the overall number of aviation accidents due to the increased volume of air traffic. Therefore, reduction in the aviation accident rate is a high priority within the aviation community.

The Vehicle Systems Safety Technologies Project (VSST) of the NASA Aviation Safety Program provides knowledge, concepts and methods to avoid, detect, mitigate and recover from hazardous flight conditions, and to maintain vehicle airworthiness and health. To achieve these goals significant attention is given to the development of new sensor technologies and assessment of such hardware's performance and reliability. Development and performance evaluation of reliable sensor systems capable of operating in harsh engine environments represents one of the focused efforts of the VSST Project. The Vehicle Integrated Propulsion Research (VIPR) series of engine tests, incubated in the VSST, is a means to test and evaluate emerging health management technologies in an aircraft engine environment. The VIPR testing includes both nominal and seeded fault engine operating scenarios, and enables evaluation of engine health management sensors and diagnostic algorithms. On-engine evaluation is a critical step in order for Integrated Vehicle Health Management (IVHM) technologies to mature from laboratory work and simulations to demonstrations needed for industry and military acceptance.

Among the sensors included in the VIPR engine tests is a fiber optic temperature sensor developed at NASA Glenn Research Center (GRC). The sensor itself is a fiber Bragg grating (FBG) formed by variations in the refractive index of the fiber core. Fiber optic sensors are immune to electromagnetic interference, chemically stable, and have small size and weight. Changes in any of the parameters of light propagating in optical fibers, light intensity, polarization, and wavelength, can be used in sensing systems for measurement purposes. However, since FBG-based sensors use variations in the wavelength of light that propagates through them as means to conduct the sensing, the resultant signals are immune to incidental light variations in the optical fiber. That feature makes the temperature measurements acquired by FBGs potentially much more stable and reliable than those acquired by conventional measurement techniques.

This paper, in addition to explaining the basic principles of fiber Bragg gratings, describes briefly various types of FBGs and focuses on the GRC-developed process to manufacture high temperature FBG-based sensors. The paper also describes the considerations that played a role in the design, construction, and installation of the sensor. The paper culminates with a description of the VIPR tests and analysis of the results.

Testing of fiber optic temperature sensors as a part the VIPR effort permits, for the first time, the placing of FBG-based sensors in engine environments. Successful test results elevates the technology development levels and help to understand any technology gaps that may limit the insertion of this new sensing technology into an aircraft engine.

\section{Theory and Operation of Fiber Bragg Gratings}

Fiber Bragg gratings are passive fiber optic components commonly used in the communication industry for spectral filtering and re-routing of optical signals. FBGs are small in dimension, relatively low cost and exhibit good immunity to external electromagnetic interference and harsh ambient conditions. They have replaced bulk optic mirrors and beam splitters in equipment, which increases system stability and portability. In-depth theoretical 
analyses of the electromagnetic wave propagation through periodic structures in general ${ }^{1-3}$ and FBGs in particular ${ }^{4-6}$ can be found elsewhere.

A typical FBG consists of a longitudinal periodic variation of the index of refraction in the core of an optical fiber. The principle of operation of an FBG is illustrated in Fig. 1.

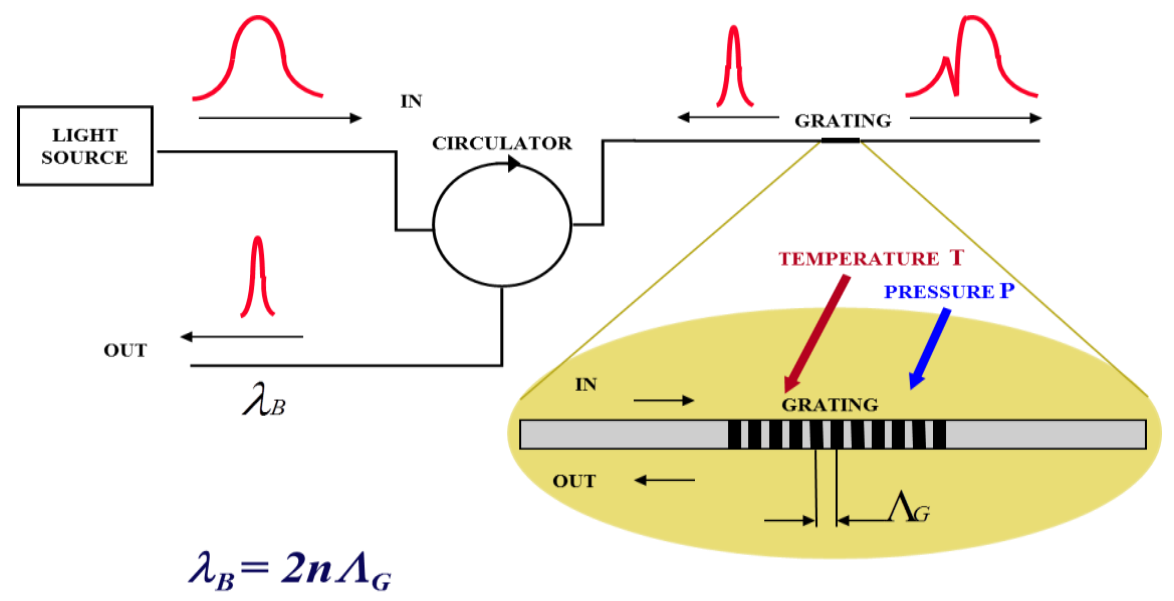

Figure 1. Schematics of operation of fiber Bragg grating sensor system.

As a broad spectrum of light propagates through a fiber with an FBG a small portion of that spectrum is reflected back by the grating. The central wavelength of that portion of the spectrum is the Bragg wavelength, $\lambda_{B}$, and is determined by both the grating line spacing $\Lambda_{G}$ and the effective refractive index $n$ :

$$
\lambda_{B}=2 n \Lambda_{G}
$$

The strength of the light at the Bragg wavelength that reflects from the grating depends on the number, $N$, of individual gratings with the line spacing $\Lambda_{G}$, effective refractive index, $n$, and amplitude of the periodic variations of the core refractive index, $\Delta n$. An approximate expression for the full-width-half-maximum bandwidth if a grating is shown below ${ }^{7}$ :

$$
\Delta \lambda=\lambda_{B} S \sqrt{\left(\frac{\Delta n}{2 n}\right)^{2}+\left(\frac{1}{N}\right)^{2}}
$$

Parameter S in Eq. (1) assumes values from 0.5 to 1.0 and depends mainly on the type of apodization used to minimize the power loss in the side lobes of the FBG spectrum ${ }^{8}$. Value 1.0 is used for the strongest gratings.

Any changes in the environment in which the grating is placed into lead to changes either in the grating line spacing, $\Lambda_{G}$, or effective refractive index, $n$, or both, and consequently in the Bragg wavelength, $\lambda_{B}$.

Thus, for instance, the effect of strain and temperature on the shift in the Bragg wavelength can be expressed using the following equation"

$$
\Delta \lambda_{B}=2 n \Lambda_{G}\left[\left(1-\left(\frac{n^{2}}{2}\right)\right)\left(P_{12}-v\left(P_{11}+P_{12}\right)\right)\right] \varepsilon+\left[\alpha+\frac{d n}{d \mathrm{~T}} / n\right] \Delta \mathrm{T}
$$

Since $\Delta \lambda$ is also a function of refractive indexes, their change with temperature would also lead to changes in the bandwidth of the FBG spectrum.

\section{Design, Manufacturing, and Calibration of HTFOS}

Fiber Bragg gratings are formed in silica fibers with photorefractive dopants during the exposure of the fibers to ultraviolet light ${ }^{10}$. Usually the dopant is germanium oxide. However other materials doped into silica have been reported to have similar responses to such exposures. 


\section{A. High Temperature Fiber Bragg Gratings}

Various types of FBGs have been developed over the last decade with various performance characteristics at elevated and high temperatures. The gratings have also been grouped into two major categories based on the way they are manufactured ${ }^{11,12}$. One category identified as Type I gratings includes gratings manufactured by exposure of fiber doped with photorefractive material to moderate levels of either continuous or pulsed illumination of ultraviolet radiation. That category has several sub-categories that depend on the dopant levels, hydrogen loading, presence of co-dopants, and nuances of the manufacturing process. The other category, called Type II gratings, is produced by exposing fibers to very short pulses with intensity that exceeds the damage threshold. A combination of processes that involve photorefractive changes in the fiber with high intensity femtosecond laser pulsing has also been reported.

Among various types of fiber Bragg gratings, only two result in FBGs capable of withstanding temperatures on the order of $1000{ }^{\circ} \mathrm{C}$. One type includes regenerated gratings ${ }^{13}$ which are a variation of Type I gratings and the other one includes Type II FBGs.

Over the past decade, NASA Glenn has been involved in the development of high temperature sensors for engine applications using regenerative gratings. During that time, NASA Glenn developed a process to manufacture regenerative FBGs with high thermal stability by exposing Type I gratings to a slow annealing to temperatures above $900^{\circ} \mathrm{C}^{14}$.

\section{B. Construction and Laboratory Calibration of Regenerating Gratings}

Construction of a high temperature fiber optic sensor, HTFOS, involves design, packaging, thermal annealing, and in-house post-annealing testing and calibration. The first and second stages of the process are depicted in Fig. 2. The optical fiber has a polyimide coating capable of withstanding temperatures up to $300^{\circ} \mathrm{C}$. A set of two ceramic tubes is used to construct a robust sensor unit with a smaller diameter tube being placed inside a larger one. The smaller tube has an outer diameter $d_{l}$ and inner diameter sufficient to accommodate the polyimide coated fiber. The fiber with an FBG is inserted in the smaller diameter tube in such a way that the grating is located close to one end of the tube. The grating is Type I grating written into a germane-silicate fiber after subjecting the fiber to hydrogen loading at a couple thousand psi for several days. A larger tube has outer diameter $d_{2}$. Diameters $d_{1}$ and $d_{2}$ are selected to accommodate the fiber optic (FO) connector. The length of the smaller diameter tube is $l_{l}$ and the larger diameter tube length is $l_{2}$. The smaller diameter tube protrudes beyond the larger tube by a length of $\left(l_{1}-l_{2}\right)$. At the end of the process the FBG is located inside that protrusion. The FO connector is a commercial grade FC/APC connector placed and secured at the other end of the sensor unit using a commercial grade high temperature optical epoxy.

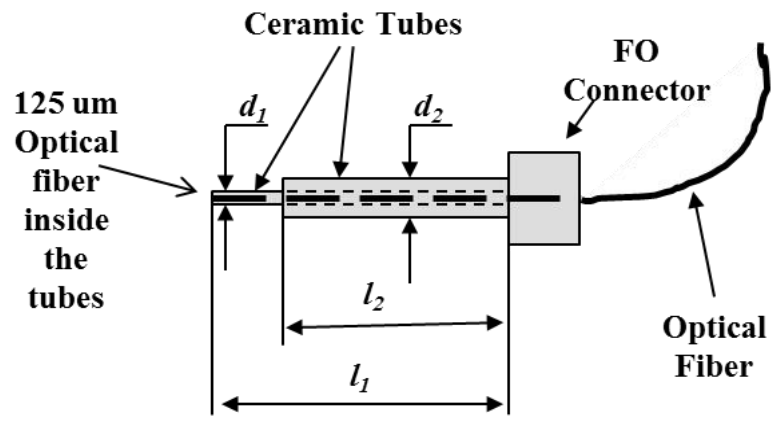

a)

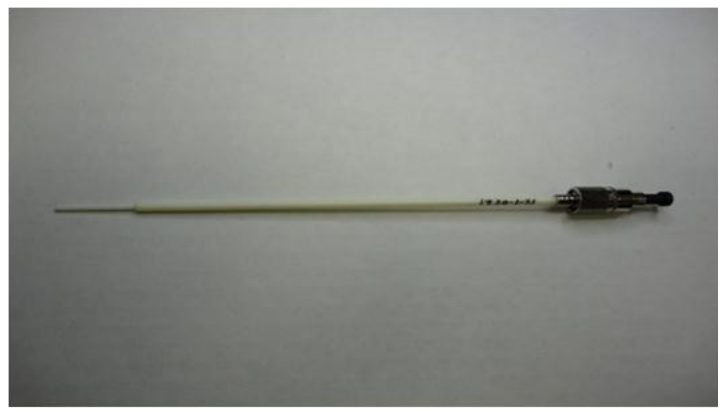

b)

Figure 2. First two stages of the HTFOS construction process: $a$ ) design and $b$ ) packaging.

Once the HTFOS sensor was packaged, the annealing is conducted using a tube furnace. The tip of the packaged sensor is inserted through the port in the back of the furnace in such way that just the end of the sensor unit is exposed to annealing temperatures up to about $1000^{\circ} \mathrm{C}$. The process is monitored to assure formation of a regenerated grating. Figure 3 shows typical changes in the FBG reflectivity through the annealing process. 


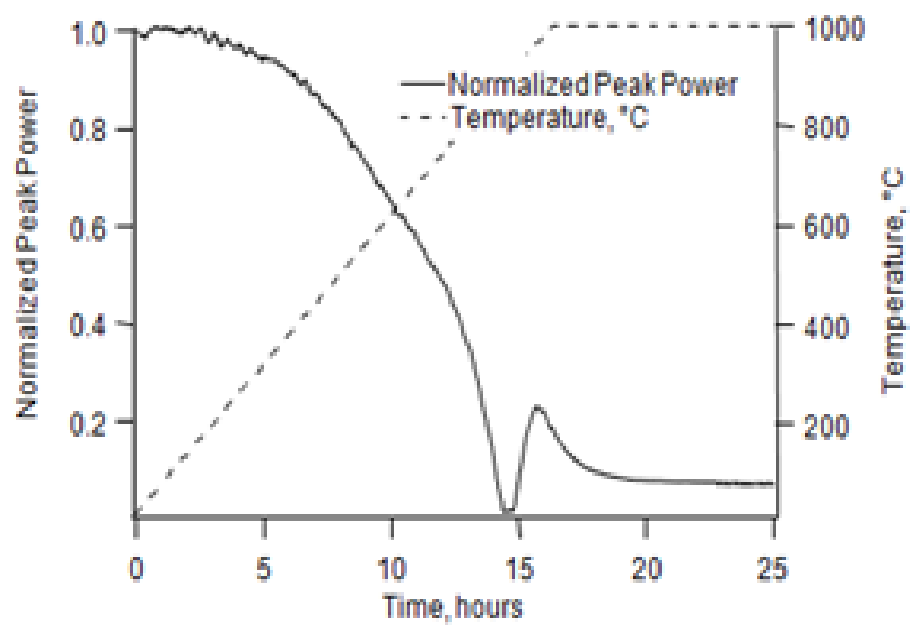

Figure 3. Characteristic FBG reflectivity profile associated with annealing to produce regenerated FBG.

The last step in the manufacturing process is post-annealing testing and calibration. The post-annealing testing is performed at maximum temperatures $150-250^{\circ} \mathrm{C}$ below the annealing temperatures to avoid any additional annealing of the sensor unit. Each HTFOS unit underwent exposure to a continuing increase of temperature from room temperature to the maximum of $800^{\circ} \mathrm{C}$. After reaching $800^{\circ} \mathrm{C}$ the unit was also exposed to one thermal cycling down to $500^{\circ} \mathrm{C}$ and back up to $800^{\circ} \mathrm{C}$. After that the unit was permitted to cool down to room temperature. The thermal profile of post-annealing testing is shown in Fig. 4. The red line in Fig. 4a represents the temperature the HTFOS was subjected to as a function of time. The black line in that figure represents the corresponding values of the Bragg wavelength, $\lambda_{G}$. Figure $4 \mathrm{~b}$ represents the hysteresis observed while the HTFOS unit was thermally cycled between 500 and $800^{\circ} \mathrm{C}$.

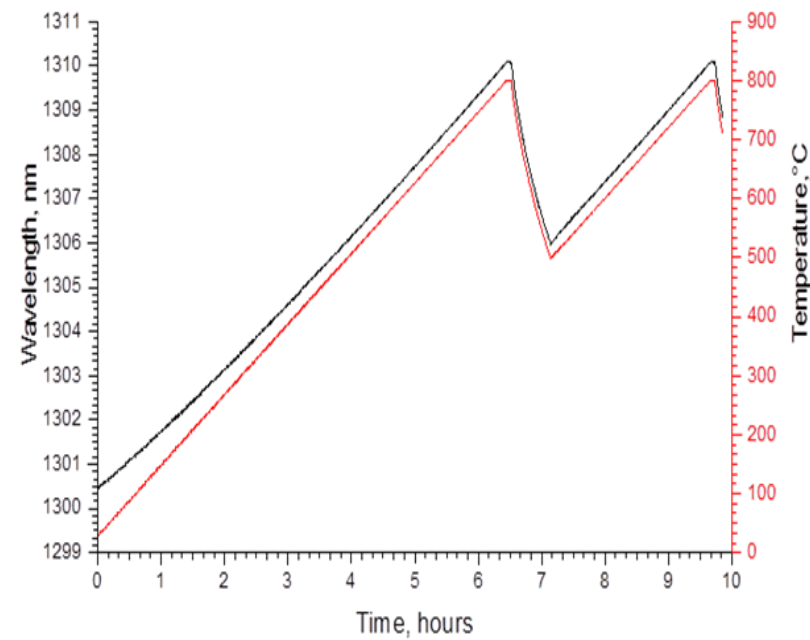

a)

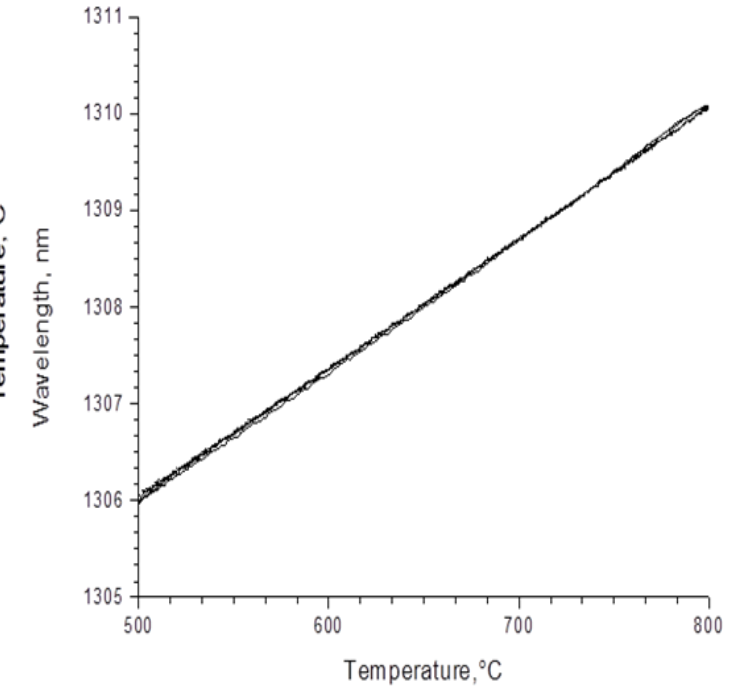

b)

Figure 4. Post annealing testing of HTFOS: $a$ ) thermal profile; b) hysteresis observed.

Analysis of curves presented in Fig. 4 show that the calibration appears fairly linear over a range of temperatures from $350^{\circ} \mathrm{C}$ to $750^{\circ} \mathrm{C}$. However, in order to extend the calibration curve fit to a temperature range from $150^{\circ} \mathrm{C}$ to 
$800^{\circ} \mathrm{C}$, a third order polynomial fit was used. Both curve fits (linear and third order polynomial) are shown with in Fig. 5. The third order polynomial calibration curve was ultimately used in the analysis of the VIPR2 test data.
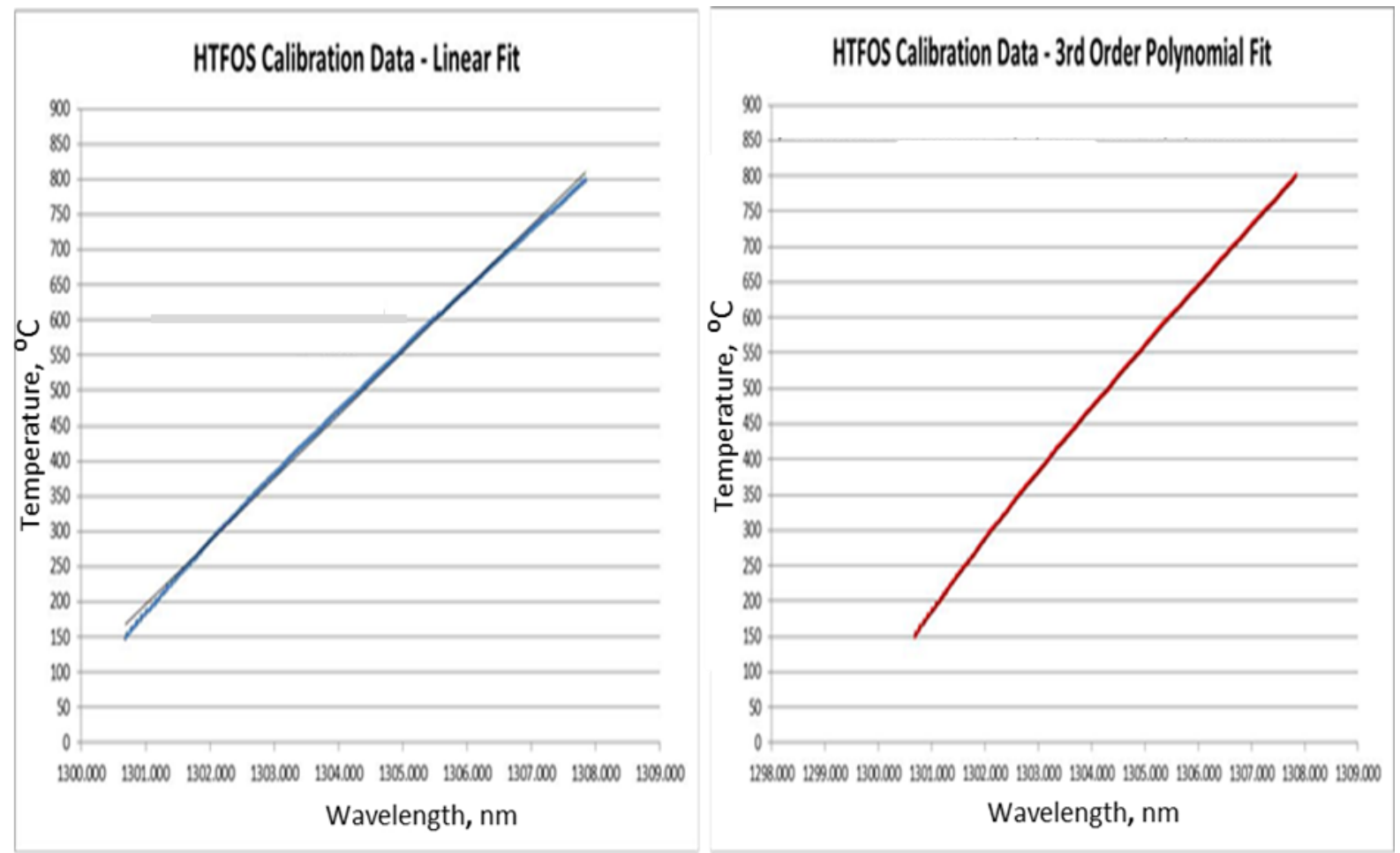

Figure 5. Calibration curves for HTFOS sensor with the linear fit (left) and third order polynomial fit (right).

\section{Field Installation and Testing of HTFOS}

An HTFOS system was developed at the NASA GRC specifically for the inclusion in the second phase of the VIPR engine tests, VIPR 2, that were performed at the NASA Armstrong Flight Research Center, AFRC VIPR is a series of ground -based engine tests conducted on an Air Force Boeing C17 aircraft equipped with Pratt \& Whitney F117 turbofan engines. During VIPR 2 testing, the HTFOS was not installed on engine, but was instead installed on a rig located behind the engine know as Emission Sensing System, ESS, rig. This enabled the HTFOS to measure the hot gas temperatures exiting the engine.

\section{A. Specific Design Considerations}

The design of the HTFOS intended for the engine tests was done with specific considerations in mind. First, since the sensor unit was to be installed behind the engine with a significant part of the unit exposed to the high temperature exhaust plume the length of the entire unit was about 24". Such length permitted placing the temperature sensitive conventional optical connector away from the tip of the sensor which was designed to see engine exhaust operational temperatures. Furthermore, since the sensor's optical connector was attached to the optical fiber by an epoxy that has a working temperature limit of $200^{\circ} \mathrm{C}$ special measures were taken to protect the connector by adding heat shielding sleeves.

Second, because the sensor tip was to be positioned at the height of the engine exhaust the entire HTFOS had to be incorporated in the upper mast of the ESS rig. The ESS rig itself consisted of two vertical masts, upper and lower, placed on a movable platform. The movable platform also housed special translation stages to move both masts in the plane normal to the direction of the engine exhaust plume. Figure 6 shows schematically the location of the HTFOS (left) on the ESS rig behind the engine. Also shown is a schematic of the ESS rig. 


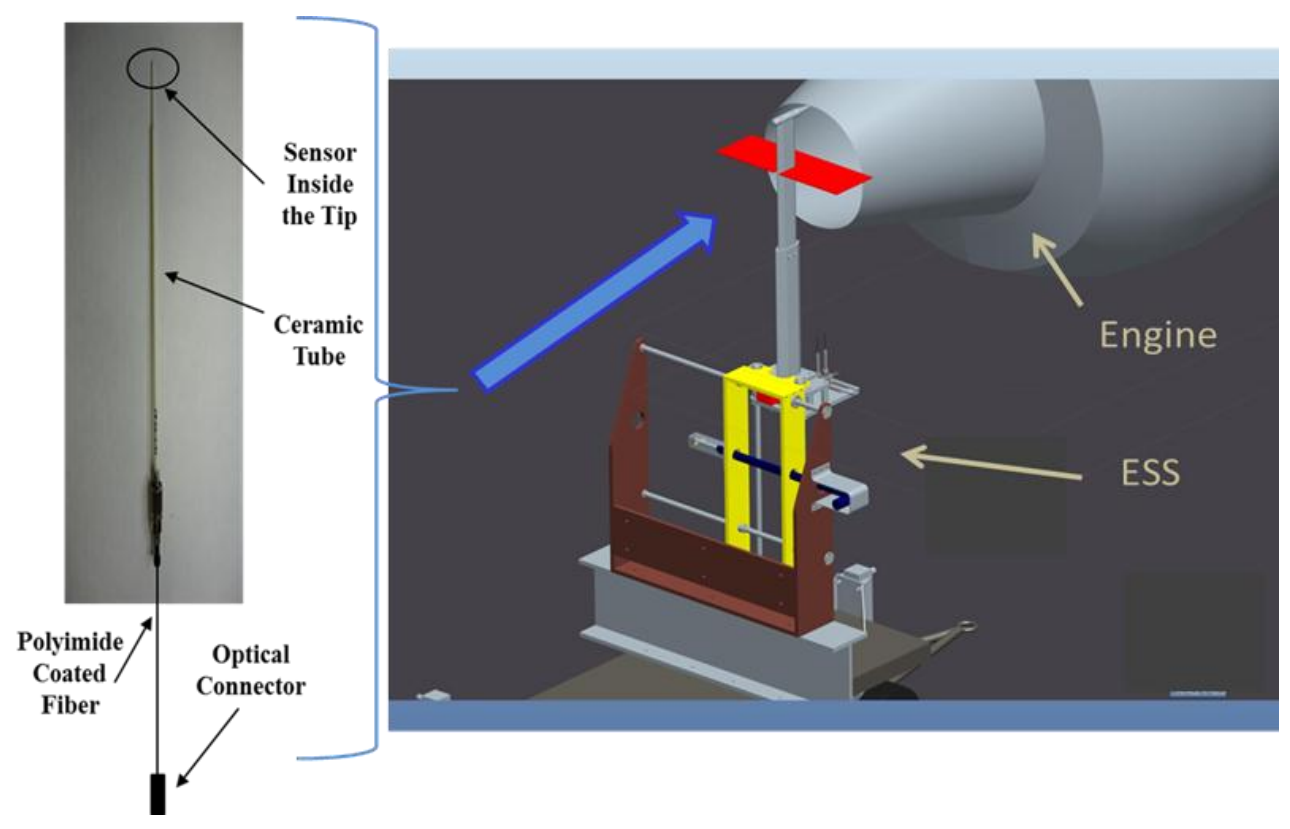

Figure 6. Schematics showing the location of HTFOS (left) on the ESS rig behind the engine.

The top of the upper mast had a bullet shaped head designed to accommodate pipes and hoses for gas emission sensing. The HTFOS unit was fastened to a specially designed holding plate that fit inside the upper mast in a way that only the 0.5 " long tip of the sensor unit was protruded above the head. That tip of the sensor unit was exposed directly to the hot gases exhausted from the engine during the tests.

Figure7 shows pictures of the HTFOS unit installed inside the upper mast, Fig. 7a, and the sensing tip of the unit protruding above the bullet-shaped head of the upper mast after its installation, Fig. 7b.

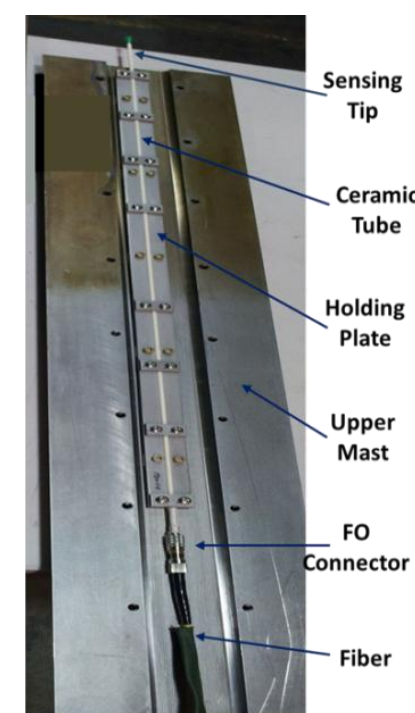

a)

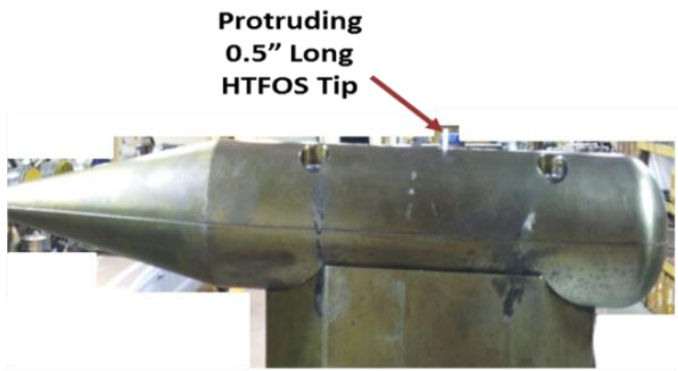

b)

Figure 7. Pictures of HTFOS unit:

a) unit installed inside the upper mast;

b) tip of the unit protruding above the bullet-shaped head of the upper mast. 
The fiber at the bottom of Fig. 7a is shown protected by a heat protecting sleeve. The sensing tip of the HTFOS is seen in Fig. $7 \mathrm{~b}$ as a thin white stick prodruding for about 0.5 " above a massive bullet-shaped metal structure.

\section{B. Signal processing}

The HTFOS signal processing system was placed in a remotely located trailer and the data transmission and collection from the HTFOS to the signal processing system was done via a fiber optic cable. The signal processing system included a superluminescent emitting diode, SLED, optical distribution board, optical spectrum analyzer, OSA, and computer with the LabView software. The signal processing system and its connection with the HTFOS are shown in Fig. 8.

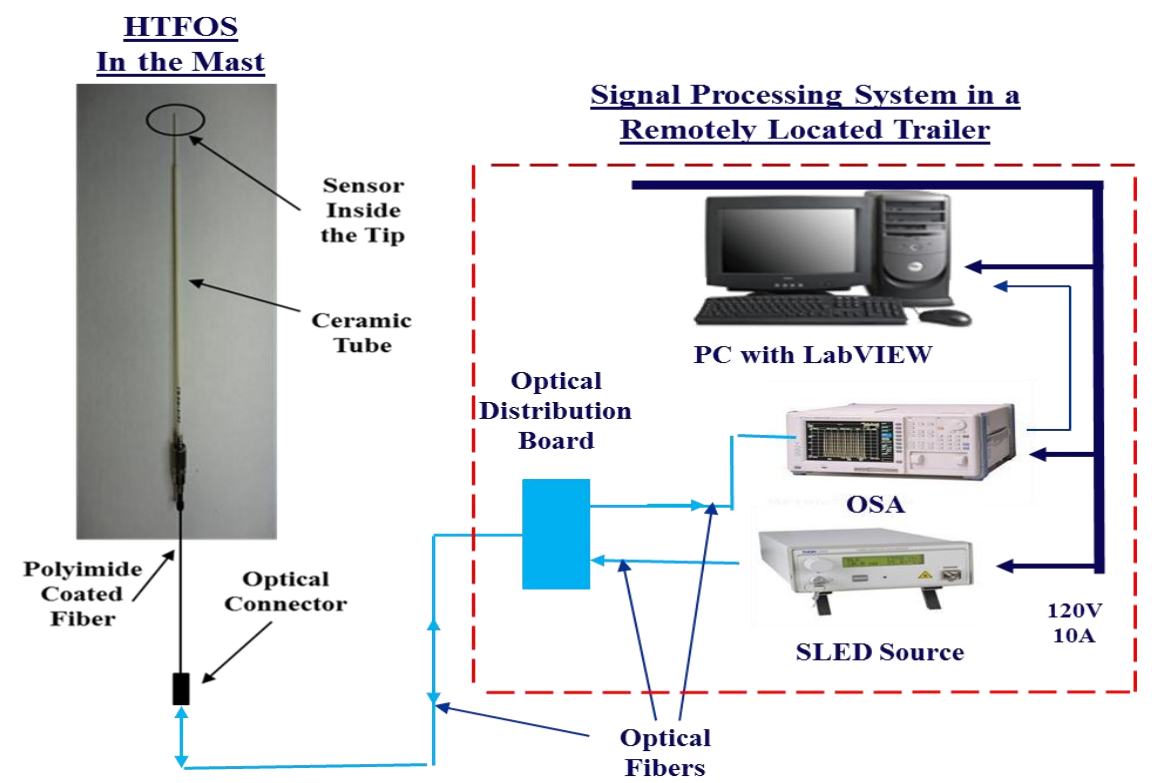

Figure 8. Schematics of the signal processing system and its connection to the HTFOS.

The SLED was a light source with a broad spectrum centered at about $1300 \mathrm{~nm}$. The light from the SLED was sent through the optical distribution board and optical fibers toward the HTFOS secured in the upper mast of the ESS. The optical distribution board was an assembly of single mode fiber optic components designed to send most of the optical power emitted by the SLED to the HTFOS and redirect most of the returned optical signal to the OSA. The OSA which normally displayed the spectrum of a detected signal was controlled by computer with the LabView software. The LabView software processed and recorded the signals detected by the OSA.

\section{Engine Tests}

The HTFOS acquired data over 3 days of VIPR 2 engine tests. During the tests, the ESS rig was positioned behind the engine as approximately depicted in Fig. 9. The HTFOS was installed inside the ESS rig and protruding about 0.5 " above the bullet-shaped top of the mast. During VIPR 2 testing the engine was operated over a range of power settings which resulted in temperature changes of exhaust gases. The temperature of the exhaust gases is measured by exhaust gas temperature, EGT, sensors installed in the engine. The HTFOS was also recording the temperature readings of the exhaust gases through the values of the Bragg wavelength reflected by the FBG. After completion of the tests the data from the HTFOS were processed and compared with the corresponding EGT data. 


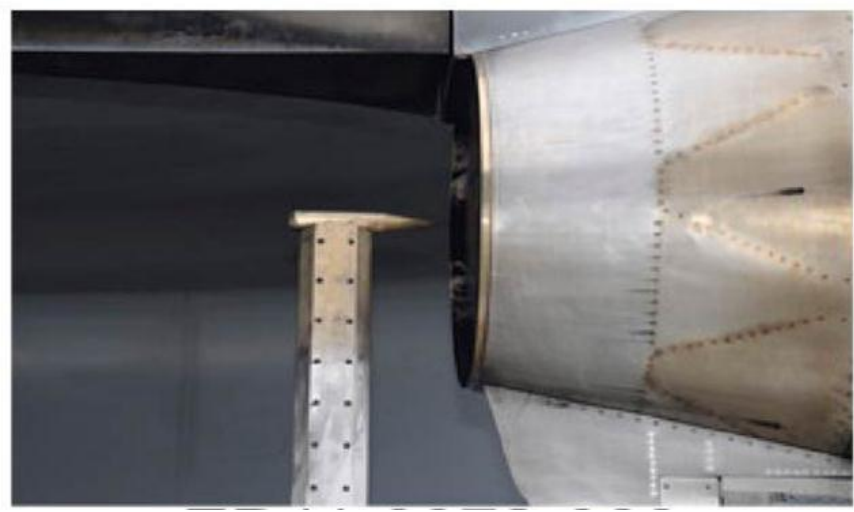

Figure 9. Approximate position of the ESS rig behind the engine during the tests.

The first engine run during VIPR 2 tests was a preservation oil burn-off run. That process occurs when the engine is run for the first time post-overhaul. This produces a copious amount of oil vapor during the oil burn-off. Since the HTFOS system had its own power source that was not dependent upon the aircraft's power systems, the HTFOS sensor was powered on for about a half hour before the engine test began. The changes in temperature read by the sensor were detected as changes in the wavelength. During the post-test data processing the wavelength readings were converted to temperature and compared with temperature readings from the EGT sensors. Figure 10 shows the results. Figure 10a on the left shows readings from the HTFOS during a period of time that included the time the engine was running during the preservation oil burn-off. The engine run time is indicated on the figure by shaded section. To the right, in Fig. 10b, the corresponding readings from the EGT sensors are presented.

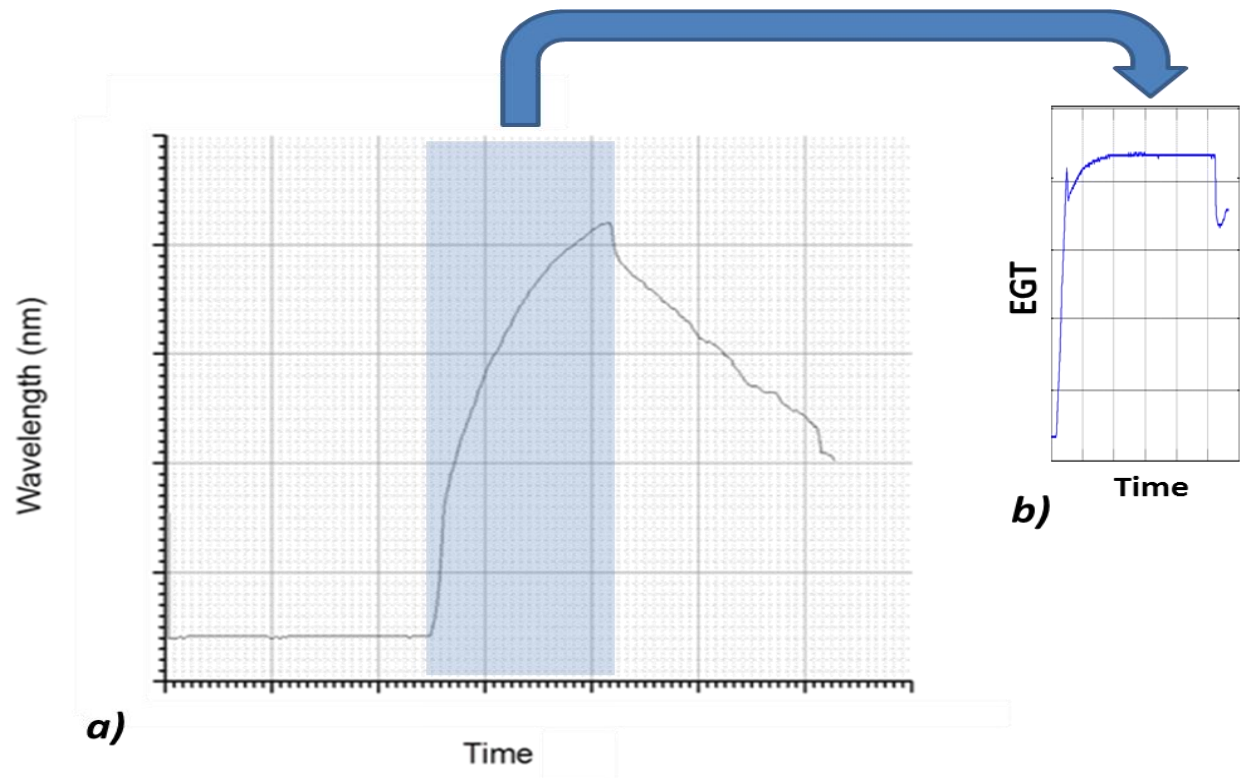

Figure 10. Measurements acquired during the preservation oil burn-off run: a) wavelength readings from the HTFOS, b) temperature readings from the EGT sensors.

The comparison of results from the HTFOS and EGT shows that the HTFOS data exhibit a significantly slower response to a rapidly changing temperature. That is attributed to a presence of the massive bullet-shaped top of the ESS rig upper mast which acted as a thermal buffer preventing the HTFOS from responding fast to changes in engine exhaust gas temperature. The HTFOS also provided temperature data for the entire duration of the test including the time prior to engine start and after engine shutdown. Furthermore, the HTFOS sensor operation was found to be uninhibited by the oil burn-off process. 
The second and third days of VIPR 2 testing entailed operating the test engine under both nominal and faulty operating scenarios. The evaluated fault scenarios include seeded faults manually introduced into the engine's station 2.5 bleed valve and $14^{\text {th }}$ stage bleed valve. During the tests, engine measurement data were acquired while the engine was operated over a range of power settings. The EGT and HTFOS measurement data acquired on the second and third days of VIPR 2 testing are shown in Figs. 11a and 11b, respectively.

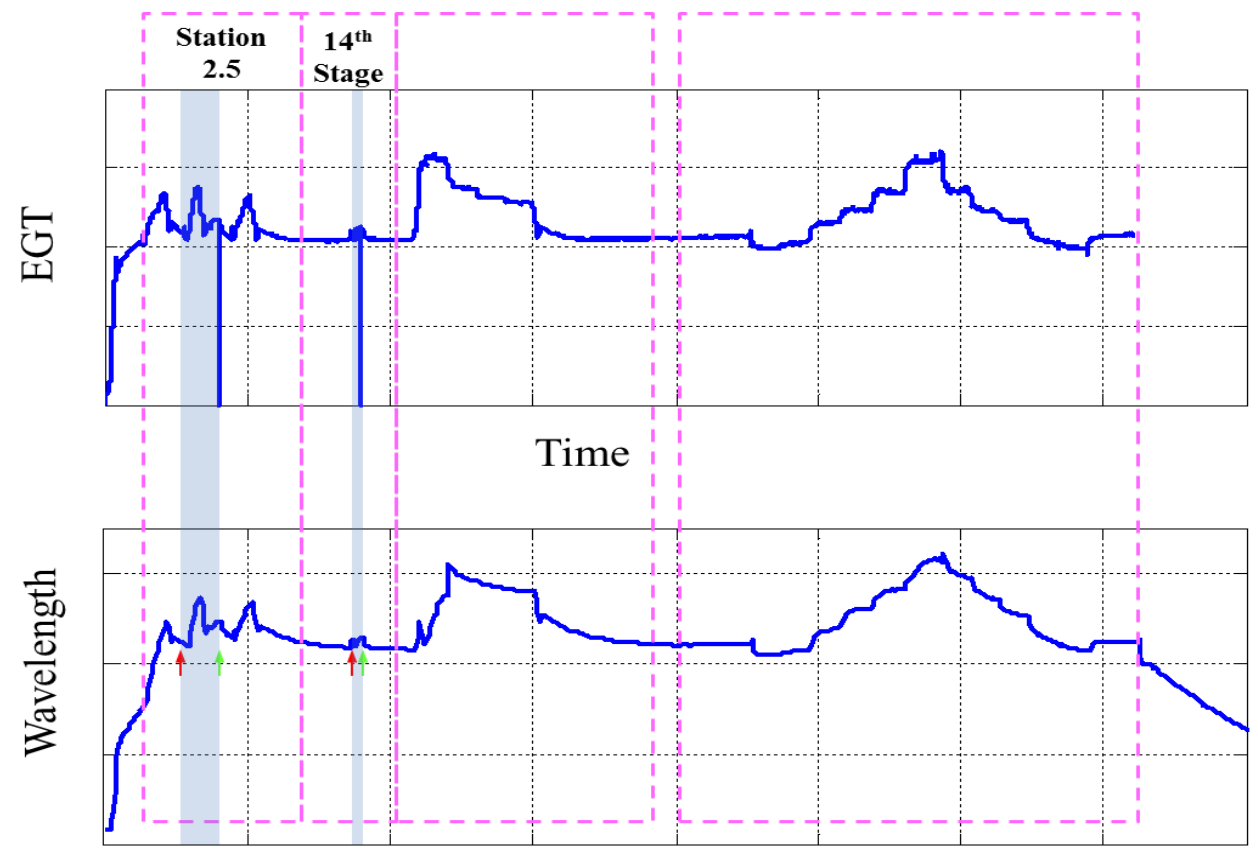

a)

Time

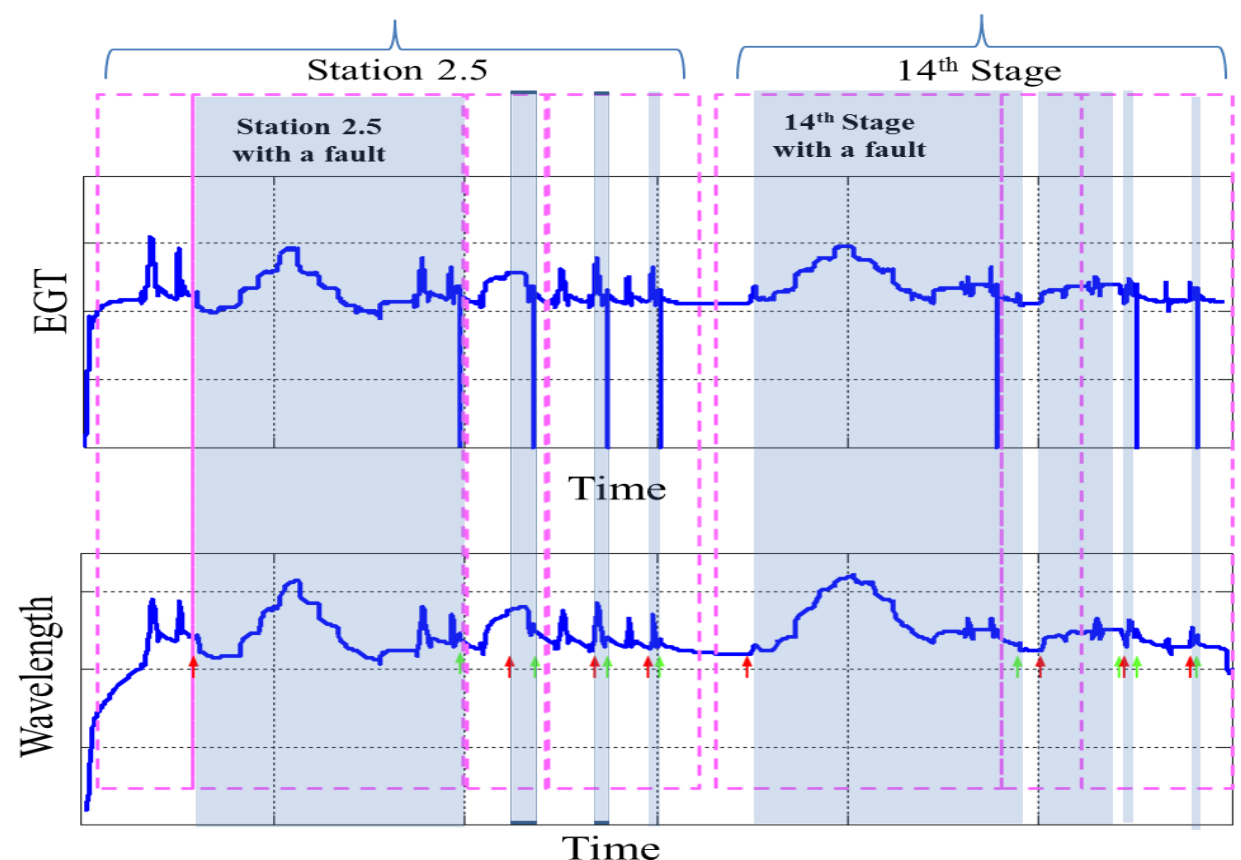

b)

Figure 11. Temperature obtained from the EGT (upper plots) and the HTFOS (lower plots) during the $2^{\text {nd }}($ plot $a)$ and $3^{\text {rd }}$ (plot $b$ ) days of VIPR 2 testing. 
The upper plots in both figures represent the EGT temperature readings and the lower plots show the corresponding wavelength readings from the HTFOS. Also, red arrows on the lower plots indicate an introduction of a bleed fault and the green arrows indicate its removal. The time intervals between the introduction and removal of the faults are approximately indicated by shaded areas.

The results obtained during the $2^{\text {nd }}$ and $3^{\text {rd }}$ days of testing have shown a good similarity between the two plots corresponding to the temperature readings from the EGT and the wavelength readings from the HTFOS. The HTFOS has also shown a similar responsivity to the introduction and removal of faults as the EGT sensors do. It has responded properly to fault insertions and removals in both the station 2.5 bleed valve and the 14th stage bleed valve. During the normal operating scenarios the HTFOS responses to changing engine operating temperatures are also similar to those acquired by the EGT sensors.

Somewhat slower responses of the HTFOS to rapid changes in the engine operating conditions are also observed, especially in the "stair-step" sections of the plot. As was mentioned earlier in the paper, this slower dynamic response could be attributed to the thermal mass of the massive bullet-shaped top of the upper mast. Furthermore, HTFOS wavelength plots exhibit some hysteresis (i.e., measured values are different stepping up in power vs. stepping down in power for same fan speed settings). That may be caused by the thermal mass of the sensor unit itself.

\section{Conclusion}

The VIPR 2 testing of the high temperature fiber optic sensor (HTFOS) in real engine exhaust environments was highly successful. These tests demonstrated that the HTFOS was capable of surviving the engine exhaust plume environment and tracked the exhaust temperature very well. The HTFOS, through the wavelength of light reflected by the grating, was able to detect changes in the exhaust gas temperature. Furthermore, during the post-test processing the changes in the wavelength were correlated to the corresponding changes in engine operating condition. The results of the tests also showed that the HTFOS temperature data compared well with the data from EGT sensors.

The tests have enabled a step forward in the development of HTFOS technology. Also, they have helped to identify technology gaps and future technology development needs. One of them is the need to miniaturize the sensor holding structure. However any efforts to make the ceramic rods that house the fiber shorter will bring the optical connector and the rest of the system closer to the hot area where the sensing tip is located. During the VIPR 2 tests this issue was avoided by making the length of the fiber holding ceramic rod sufficiently long. The length of rods used during the tests was about 20 inches. That design removed the connector at the end of the rod and the fiber attached to it from the hot zone and put it in a relatively benign thermal environment. The future development of the HTFOS should include miniaturization and proper design of the sensor structure to assure a thermal stability of all components in the high temperature zone.

Another near term gap is miniaturization of the signal processing electronics and making it compatible with the aircraft environment. Those technology gaps should be addressed in the design of HTFOS systems for future onengine applications.

\section{Acknowledgments}

The work has been supported by the Vehicle Systems Safety Technologies (VSST) Project of NASA's Aviation Safety Program. The authors want to acknowledge the NASA Armstrong Flight Research Center personnel for their support during the preparatory and implementation stages of the tests. The authors also want to express special gratitude to Mr. Donald Simon, research engineer in the GRC Intelligent Control and Autonomy Branch, for helping to interpret the temperature measurement readings acquired from both the EGT sensors and the HTFOS due to changing engine operating conditions.

\section{References}

\footnotetext{
${ }^{1}$ Yeh, P., Yariv, A., and Hong, C.-S., "Electromagnetic propagation in periodic stratified media. I. General theory," J. Opt. Soc. Am., Vol. 67, No. 4, 1977, pp. 423-438.

${ }^{2}$ De Vre, R. and Hesselink, L., "Diffraction analysis of layered structures of photorefractive gratings," J. Opt. Soc. Am. A, Vol. 13, No. 2, 1996, pp. 285-295.
} 
${ }^{3}$ Winick, K. A., "Effective-index method and coupling-mode theory for almost-periodic waveguide gratings: a comparison," App. Opt., Vol. 31, No. 6, 1992, pp. 757-764.

${ }^{4}$ Hill, K. and Meltz, G., "Fiber Bragg Grating Technology Fundamentals and Overview," J. Light. Tech., Vol. 15, No.8, 1997, pp. 1263-1276.

5 Zhao, Y. and Plais, J. C., "Fiber Bragg Grating Coherence Spectrum Modeling, Simulation, and Characteristics," J. Lightwave Techn., Vol. 15, No. 1., 1997, pp. 154-161.

${ }^{6}$ Batiato, J. M. and Hulick, K. E., "Fiber Optic Bragg Gratings," AFRL-SN-RS-TR-1998-179, 1998.

${ }^{7}$ Russell, P. St. J., Archambault, J.-L., and Reekie, L., "Fibre gratings,” Physics World, October 1993, pp. 41-46.

${ }^{8}$ Ugale, S. and Mishra, V., "Fiber Bragg Grating Modeling, Characterization and Optimization with Different Index Profiles," Int. Journal of Engineering Science and Technology, Vol. 2, No. 9, 2010, pp. 4463-4468.

${ }^{9}$ Othonos, A., "Fiber Bragg gratings," Rev. Sci. Instrum., Vol. 68, No. 12, 1997, pp. 4309-4341.

${ }^{10}$ Hill, K. O., Malo, B., Bilodeau, F., and Johnson, D. C., "Photosensitivity in Optical Fibers," Annu. Rev. Mater. Sci., Vol. 23, 1993, pp. 125-157.

${ }^{11}$ Canning, J., "Fiber gratings and devices for sensors and lasers," Laser \& Photon. Rev., Vol. 2, No. 4, 2008, pp. $275-289$.

${ }^{12}$ Mihailov, S. J., "Fiber Bragg Grating Sensor for Harsh Environments," Sensors, Vol. 12, 2012, pp. 1898-1918.

${ }^{13}$ Canning, J., Bandyopadhyay, S., Biswas, P., Aslund, M., Stevenson, M., and Cook, K., "Regenerated Fibre Bragg Gratings," Frontiers in Guided Wave Optics and Optoelectronics, edited by B. Pal, [online publication], Chapter 18, URL: http://cdn.intechopen.com/pdfs-wm/8443.pdf [cited 25 March 2014].

${ }^{14}$ Adamovsky, G., Bramante, R., Holguin, A., Chan, H., Mackey, J., and Floyd, B., "Fiber Bragg Based Optical Sensors for Extreme Temperatures," AIAA Infotech@Aerospace 2011 Conference, 29-31 March 2011, St. Louis, Missouri, AIAA $2011-1576$. 